\title{
Solutions of Gross-Pitaevskii equations beyond the hydrodynamic approximation: Application to the vortex problem.
}

\author{
Vladimir V. Konotop* \\ Departamento de Física e Centro de Física da Matéria Condensada, Universidade de Lisboa, Complexo Interdisciplinar, \\ Av. Prof. Gama Pinto, 2, Lisbon, P-1649-003 Portugal \\ Víctor M. Pérez-García ${ }^{\dagger}$ \\ Departamento de Matemáticas, E.T.S.I. Industriales, Universidad de Castilla-La Mancha, \\ Avda. Camilo José Cela, 3, Ciudad Real, 13071 Spain.
}

(November 20, 2018)

\begin{abstract}
We develop the multiscale technique to describe excitations of a Bose-Einstein condensate (BEC) whose characteristic scales are comparable with the healing length, thus going beyond the conventional hydrodynamical approximation. As an application of the theory we derive approximate explicit vortex and other solutions. The dynamical stability of the vortex is discussed on the basis of the mathematical framework developed here, the result being that its stability is granted at least up to times of the order of seconds, which is the condensate lifetime. Our analytical results are confirmed by the numerical simulations.
\end{abstract}

PACS number(s): 03.75.-b, 03.75.Fi, 67.57.Fg

\section{INTRODUCTION}

Since the remarkable experimental realization [1] of the Bose-Einstein condensation (BEC) there has been an explosion of the experimental and theoretical activity devoted to the physics of dilute ultracold bosonic gases [2].

From the theoretical point of view, many of current condensate systems are cold enough that the mean field theories can be applied to describe the ground state of the system [3]. The most classical model arising in this context for the order parameter of the system is the the Gross-Pitaevskii (GP) equation. In fact, many relevant features of the condensate such as free expansion [4], low energy excitations [5], collapse [6. [], dynamics of multicomponent systems [8], vortex generation and dynamics [9.10], and many others, can be understood within the framework of this "simplest" mathematical model.

Mathematically, the GP equation, to be written explicitly below, is an equation of Nonlinear Schrödinger type. These equations have been studied extensively both in the physical and mathematical literatures [12, 13] since they provide an universal model for the study of the dynamics of envelope waves. Some fields where these equations arise are plasma physics [14], fiber and integrated optics [15], wave propagation in Kerr media [16], water waves [17], biomolecule dynamics 18 and fundamentals of quantum mechanics [19]. One of the distinctive features of the equation as it appears in BEC problems is the presence of an external potential -the trap-, which affects essentialy the spectrum of the "linear part", i.e. of the underlying Schrödinger equation.

The mathematical analysis of the GP equation in the situations common in BEC is very complicated and this is why most theoretical studies deal with either numerical simulations or some kind of approximations such as the ThomasFermi, the hydrodynamic approach or simply reduce the number of degrees of freedom by using the variational approach based on trial functions. To be precise let us write explicitly the equation for the order parameter, whose form is

$$
i \hbar \frac{\partial \Psi}{\partial t}=-\frac{\hbar^{2}}{2 m} \nabla^{2} \Psi+V_{e x t}(\mathbf{r}) \Psi+g|\Psi|^{2} \Psi
$$

where the external potential can be well approximated by

$$
V_{e x t}=\frac{1}{2} m\left(\omega_{x}^{2} x^{2}+\omega_{y}^{2} y^{2}+\omega_{z}^{2} z^{2}\right)
$$

${ }^{*}$ Electronic address: konotop@alf1.cii.fc.ul.pt

${ }^{\dagger}$ Electronic address: vperez@ind-cr.uclm.es 
and $g=4 \pi \hbar^{2} a_{s} / m$.

The simplest approximation that can be done to obtain information on the ground state is the so called ThomasFermi approximation, which corresponds to neglecting the kinetic energy term under the assumption that it is much less than the self-interaction energy. In the cases when the trap is smooth enough this approximation is not bad although it is not consistent, since the assumption of zero kinetic energy provides a profile that has an infinite value of that quantity.

The following level of approximation, the hydrodynamic approach, can be explained by introducing new variables $n(\mathbf{r}, t)$ and $\mathbf{v}(\mathbf{r}, t)$ through the relations

$$
\begin{aligned}
\Psi(\mathbf{r}, t) & =\sqrt{n(\mathbf{r}, t)} e^{i S(\mathbf{r}, t)}, \\
\mathbf{v}(\mathbf{r}, t) & =\frac{\hbar}{m} \nabla S(\mathbf{r}, t) .
\end{aligned}
$$

Then, one arrives to the system of equations

$$
\begin{gathered}
\partial_{t} n+\nabla \cdot(\mathbf{v} n)=0 \\
m \partial_{t} \mathbf{v}+\nabla\left(V_{e x t}+g n-\frac{\hbar^{2}}{2 m \sqrt{n}} \nabla^{2} \sqrt{n}+\frac{m v^{2}}{2}\right)=0 .
\end{gathered}
$$

The conventional hydrodynamic approximation corresponds to neglecting the term proportional to $\nabla^{2} \sqrt{n}$ under the assumption that the density is a slowly varying function 24]. For a spherically symmetric potential $\left(\omega_{x}=\omega_{y}=\omega_{z}=\right.$ $\omega)$ this can be done in the limit $N a_{s} / a_{0} \gg 1$, where $a_{s}>0$ is the scattering length and $a_{0}=\sqrt{\hbar / m \omega}$ is the harmonic oscillator length, i.e. the trap size.

To the best of authors knowledge the mathematical limits of validity of the above mentioned approximations have not been established (which would imply study of the next orders of the expansion or making appropriate rigorous estimates), so far. In particular, using the estimate $n \sim 10^{12} \mathrm{~cm}^{-3}$ one finds that for $a_{s} \sim 10 \mathrm{~nm}$ the term with $\nabla^{2} \sqrt{n} / \sqrt{n}$ is much less than $g n$ for excitations whose characteristic length $\Lambda$ is of order of $10^{-3} \mathrm{~cm}$. Such a $\Lambda$ is comparable with the characteristic size $a_{0}$ of the trapping potential. Thus the question about analytical description of the excitations which vary on scales much less than $a_{0}$ appears. It is important to notice that in those cases the terms $\frac{\hbar^{2}}{m} \frac{\nabla^{2} \sqrt{n}}{\sqrt{n}}$ and $g n$ become comparable with each other and have to be treated in equivalent manner. Using the wave terminology this situation can be characterized as balance of dispersive and nonlinear effects, which should lead to stable spatially localized excitations.

It is our intention in this paper to describe an analytical tool, the multiscale approximation, which is able to provide approximate solutions of the problems under consideration in explicit analytical form. In less rigorous terms this technique has been applied in the context of BEC in Refs. [7]20] to problems with negative scattering length. The application to positive scattering length presents some new characteristics as we will see later (in particular it implies different scaling order). Specifically, we will consider a BEC in a parabolic cilindrically or spherically symmetric confining potential and obtain a set of background solutions in the form of black holes or vortices whose characteristic spatial scale is much larger than the range of inter-atomic interactions but smaller than the trap size. Working in this way we will be able not only to obtain an approximate analytical shape for the vortex solutions but also to provide results on their dynamical stability, a point which is still of great interest after the many studies devoted to it [21,22].

The outline of the paper is as follows. First, in Sec. [1] we pose the problem in precise terms. As a second step, we apply the technique of multiscale expansion to our particular problem in Sec. III. In Sec. [V] we obtain a particular set of solutions and discuss their stability. Finally, in Sec. \, we summarize our conclusions.

\section{STATEMENT OF THE PROBLEM}

We start with the three-dimensional GP equation in the form

$$
i \hbar \frac{\partial \Psi}{\partial t}=-\frac{\hbar^{2}}{2 m} \nabla^{2} \Psi+V_{e x t}(\mathbf{r}) \Psi+J_{\lambda}(\mathbf{r}) \Psi .
$$

We will preserve the integral form of the interaction term [c.f. Eq. (1.1)]

$$
J_{\lambda}(\mathbf{r})=g \int V_{\lambda}\left(\mathbf{r}, \mathbf{r}^{\prime}\right)\left|\Psi\left(\mathbf{r}^{\prime}, t\right)\right|^{2} d \mathbf{r}^{\prime}
$$


since there are situations where its role could be important [23] and it does not complicate the formalism. Here $V_{\lambda}\left(\mathbf{r}, \mathbf{r}^{\prime}\right)$ describes two-body interactions and $\lambda$ is the effective interaction length (mathematically, the size of the kernel).

The wave function is normalized to the total number of particles $N$

$$
\int|\Psi(\mathbf{r})|^{2} d \mathbf{r}=N
$$

It is reasonable to assume that the effective interaction length $\lambda$ parametrizes the kernel so that

$$
\lim _{\lambda \rightarrow 0} V_{\lambda}\left(\mathbf{r}, \mathbf{r}^{\prime}\right)=\delta\left(\mathbf{r}-\mathbf{r}^{\prime}\right) .
$$

The last limit corresponds to the usual case of local interactions and gives rise to the $|\Psi|^{2}$ nonlinear term in the GP equation [i.e. (2.1) transforms into (1.1)]. Bearing in mind this last fact we can narrow the class of the kernels under consideration by requiring

$$
V_{\lambda}\left(\mathbf{r}, \mathbf{r}^{\prime}\right) \equiv \frac{1}{\lambda^{3}} U\left(\frac{\left|\mathbf{r}-\mathbf{r}^{\prime}\right|}{\lambda}\right)
$$

where

$$
\int U\left(\left|\tilde{\mathbf{r}}-\tilde{\mathbf{r}}^{\prime}\right|\right) d \tilde{\mathbf{r}}^{\prime}=1 .
$$

and $\tilde{\mathbf{r}}=\mathbf{r} / \lambda$. As it is clear from the definition of $\lambda$, it must be of order of the scattering length:

$$
\frac{\lambda}{a_{s}}=O(1)
$$

In what follows, however we keep $\lambda$ as a parameter.

We will concentrate on a BEC in a trap with either cilindrical (with $z$-axis being the symmetry axis) or spherical symmetry, i. e. $\omega_{\alpha}=\omega$, where $\alpha=x, y$ in the former case and $\alpha=x, y, z$ in the last case. We define the characteristic scales of the condensate in the trasversal, $a_{0}=\sqrt{\hbar /(m \omega)}$, and longitudinal, $a_{z}=\sqrt{\hbar /\left(m \omega_{z}\right)}$, directions. In the case of spherical symmetry these scales coincide, $a_{z}=a_{0}$.

In order to provide a formal description of the system it is convenient to scale out the GP equation. To this end we introduce the dimensionless time and wavefuntion through

$$
\begin{aligned}
\tau & =\frac{\hbar}{2 m \lambda^{2}} t, \\
\psi(\tilde{\mathbf{r}}, \tau) & =2 \lambda \sqrt{\frac{2 \pi a_{s}}{N}} \Psi(\mathbf{r}, t) .
\end{aligned}
$$

Then the renormalized GP equation takes the form

$$
i \frac{\partial \psi}{\partial \tau}=-\Delta \psi+\tilde{V}_{e x t}(\tilde{\mathbf{r}}) \psi+\tilde{J}(\tilde{\mathbf{r}}) \psi
$$

where

$$
\begin{gathered}
\tilde{J}(\tilde{\mathbf{r}})=\int U\left(\tilde{\mathbf{r}}-\tilde{\mathbf{r}}^{\prime}\right)\left|\psi\left(\tilde{\mathbf{r}}^{\prime}, \tau\right)\right|^{2} d \tilde{\mathbf{r}}^{\prime}, \\
\tilde{V}_{\text {ext }}(\tilde{\mathbf{r}})= \begin{cases}\left(\Omega^{2} \tilde{\mathbf{r}}_{\perp}^{2}+\Omega_{z}^{2} \tilde{z}^{2}\right) & \text { cilindrical symmetry } \\
\Omega^{2} \tilde{\mathbf{r}}^{2} & \text { spherical symmetry }\end{cases}
\end{gathered}
$$

$\tilde{\mathbf{r}}_{\perp}=(\tilde{x}, \tilde{y}), \Omega=\lambda^{2} / a_{0}^{2}$ and $\Omega_{z}=\lambda^{2} / a_{z}^{2}$.

Having scaled out the kernel which describes the nonlocal interactions we have to impose the condition that the characteristic scale of the kernel variation is of order one, i. e.

$$
\left|\frac{1}{U(\tilde{r})} \frac{\partial U(\tilde{r})}{\partial \tilde{r}}\right|=O(1) .
$$

where $\tilde{r}=|\tilde{\mathbf{r}}|$ and $\tilde{\mathbf{r}}$ must be substituted by $\tilde{\mathbf{r}}_{\perp}$ in the case of the cilindrical symmetry. 


\section{MULTISCALE ANALYSIS.}

The multiscale technique is a mathematical technique for the analysis of problems where there are different spatial scales involved in the solution. The problem at hand possesses three important scales: the scattering length $a_{s}$, the trap size $a_{0}$ and the healing length $\xi=\left(8 \pi n a_{s}\right)^{-1 / 2}$. We will concentrate on the analysis of excitations of the superfluid corresponding to the scale of the healing length, which occur on top of a background with spatial scale of the order of the trap size. In other words our theory will be valid for cases when the above three parameters are related as follows

$$
a_{s} \ll \xi \ll a_{0}
$$

For the first step we recall the properties of the linear spectral problem

$$
L_{d}|n, l, m\rangle=\mathcal{E}_{n, l, m}|n, l, m\rangle,
$$

where $L_{d}=\mathcal{L}_{d}+\mathcal{M}_{d}$. The operator $\mathcal{L}_{d}$ describes the radial dependence

$$
\mathcal{L}_{d}=-\frac{1}{\rho_{0}^{d-1}} \frac{\partial}{\partial \rho} \rho_{0}^{d-1} \frac{\partial}{\partial \rho_{0}}+\Omega^{2} \rho_{0}^{2},
$$

and

$$
\begin{aligned}
& \mathcal{M}_{2}=\frac{\partial^{2}}{\partial z^{2}}+\frac{1}{\rho_{0}^{2}} \frac{\partial^{2}}{\partial \phi^{2}} \\
& \mathcal{M}_{3}=\frac{1}{\rho_{0}^{2} \sin \theta} \frac{\partial}{\partial \theta} \sin \theta \frac{\partial}{\partial \theta}+\frac{1}{\rho_{0}^{2}} \frac{\partial^{2}}{\partial \phi^{2}} .
\end{aligned}
$$

The indexes $n$ and $m$ refer to radial and magnetic quantum numbers while $l$ is the azimutal (in the spherical case) or longitudinal (in the cilindrical case) quantum number (see Appendix). Hereafter $d=2$ and $d=3$ are associated with cilindrical and spherical symmetries.

For the next consideration it is convenient to single out the radial part of the eigenfunctions and represent

$$
|n, l, m\rangle= \begin{cases}\xi_{n, m}\left(\rho_{0}\right) \zeta_{l, m}(z, \phi) & \text { cilindrical case } \\ \xi_{n, l}\left(\rho_{0}\right) \zeta_{l, m}(\theta, \phi) & \text { spherical case }\end{cases}
$$

To shorten notations in what follows we drop the second subindex of $\xi$ in the cases at hand: $\xi_{n, .} \equiv \xi_{n}$, keeping in mind that $n$ is a radial (rather than total) quantum number.

The operator $\mathcal{L}_{d}$ is considered in the space of functions satisfying the conditions

$$
\left|\xi_{n}(0)\right|<\infty, \quad \lim _{\rho_{0} \rightarrow \infty} \rho_{0} \xi_{n}\left(\rho_{0}\right)=0 .
$$

The inner product in this space is defined by

$$
\left(\xi_{n}, \xi_{n^{\prime}}\right)=\int_{0}^{\infty} \bar{\xi}_{n}(\rho) \xi_{n^{\prime}}(\rho) \rho^{d-1} d \rho
$$

Then the operator $\mathcal{L}$ is Hermitiam.

The problem at hand is directly related to the well known eigenvalue problem for the linear oscillator in two and three dimensional parabolic potential [25]. Its spectrum is discrete

$$
\mathcal{E}_{n, l, m}= \begin{cases}4 \Omega\left(n+\frac{1}{2}+\frac{m}{2}\right)+2 \Omega_{z}\left(l+\frac{1}{2}\right) & \text { cilindical case } \\ 4 \Omega\left(n+\frac{3}{4}+\frac{l}{2}\right) & \text { spherical case }\end{cases}
$$

(both cases naturally coincide after substitution $\Omega_{z} \mapsto \Omega$ and $l+m \mapsto l$ in the first formula). The orthormalized eigenfunctions of $\mathcal{L}_{d}$ are given in the Appendix.

In accordance with the multiscale method, which is a theory for weakly nonlinear problems, the solution is searched in the form of a weakly modulated linear mode. This is a main feature of the multiscale method, which means that the theory is applicable to small condensates. However, in many problems the results coming out from the multiscale expansions remain valid even for the large nonlinearity limit. Respectively we introduce a formal small parameter $\epsilon \ll 1$ and look for the solution of (2.9) in the form 


$$
\psi=\epsilon \psi_{1}+\epsilon^{2} \psi_{2}+\ldots=\left(\epsilon \xi_{n}^{(1)}+\epsilon^{2} \xi_{n}^{(2)}+\ldots\right) \zeta_{l, m}
$$

Some peculiarities of the expansion introduced are to be discussed here. First, we use the expansion of only radial part of the solution. This means that the consideration will be restricted to symmetric solutions (the generalization is straightforward but cumbersome). Second, in what follows we will concentrate on modulation of the ground state which turns out to be flat compared with the excitation itself. This however is not in contradiction with the assumption about smooth (i.e. dependent on $\rho_{1}$ ) modulation of the underlying linear mode, depending on the rapid variable $\rho_{0}$, by weak nonlinearity. This is justified by the following reasons: (i) The background solution must possess the "right" assymptotic behavior at $\rho \rightarrow \infty$, which can be obtained only by taking into account the term $\Omega^{2} \rho^{2}$ at the "linear level"; (ii) The expansion must take into account the contribution of all linear eigenmodes, rather than only the leading one [see (3.13) and discussion below]; (iii) The linear problem with the parabolic trap is solved exactly.

The functions $\xi_{n}^{(j)}$ depend on the set of spatial coordinates $\rho_{j}=\epsilon^{j} \rho$ and times $\tau_{j}=\epsilon^{j} \tau(j=0,1,2, \ldots): \xi_{n}^{(j)} \equiv$ $\xi_{n}^{(j)}\left(\rho_{0}, \rho_{1}, \rho_{2}, \ldots ; \tau_{0}, \tau_{1}, \tau_{2}, \ldots\right)$. Hereafter $\rho=\left|\tilde{\mathbf{r}}_{\perp}\right|$ in the cilindrical case and $\rho=|\tilde{\mathbf{r}}|$ in the spherical case. The variables $\rho_{j}$ and $\tau_{j}$ are regarded as independent: thus

$$
\begin{gathered}
\frac{\partial}{\partial \tau}=\frac{\partial}{\partial \tau_{0}}+\epsilon \frac{\partial}{\partial \tau_{1}}+\epsilon^{2} \frac{\partial}{\partial \tau_{2}}+O\left(\epsilon^{3}\right) \\
\frac{1}{\rho^{d-1}} \frac{\partial}{\partial \rho} \rho^{d-1} \frac{\partial}{\partial \rho}=\frac{1}{\rho_{0}^{d-1}} \frac{\partial}{\partial \rho_{0}} \rho_{0}^{d-1} \frac{\partial}{\partial \rho_{0}}+\epsilon\left(2 \frac{\partial^{2}}{\partial \rho_{0} \partial \rho_{1}}+\frac{d-1}{\rho_{0}} \frac{\partial}{\partial \rho_{1}}\right)+O\left(\epsilon^{2}\right)
\end{gathered}
$$

Before going into details of the multiscale expansion we have to clarify the physical meaning of the parameter $\epsilon$. We are interested in excitations against a background. Then in terms of the dimensionless wave function $\psi_{1}$ the normalization condition (2.3) leads to the estimate $N \sim \frac{\epsilon^{2} V}{8 \pi a_{s} \lambda^{2}}$ where $V=a_{0}^{d} a_{z}^{3-d}$ is the volume of the condensate which is limited by the confining potential. These estimates imply the normalization condition

$$
\int\left|\psi_{1}(\tilde{\mathbf{r}}, \tau)\right|^{2} d \tilde{\mathbf{r}}=\frac{a_{0}^{d} a_{z}^{3-d}}{\lambda^{d}}
$$

(notice that in the case of cilindrical symmetry, taking $\lambda=a_{s}$ one obtains that the new wave function is normalized to one). In other words the effective small parameter of the multiscale expansion can be identified as

$$
\epsilon=O\left(\sqrt{n a_{s}^{3}} \lambda / a_{s}\right)=O\left(\sqrt{n a_{s}^{3}}\right)
$$

where $n=N / V$ is the density of the condensate and 2.7) is used. Taking into account that typically $n a_{s}^{3}=$ $O\left(10^{-4} \div 10^{-6}\right)$ one concludes that $\epsilon\left(\sim 10^{-2} \div 10^{-3}\right)$ can indeed be used for the multiscale expansion. We notice that the approximation of the local kernel, $\lambda \rightarrow 0$ is evidently a particular limit of the theory. Formally, however, it is more convenient to keep $\epsilon$ as a small parameter without specification and substitute it by one in the final formulas.

We are interested in the evolution of an initially unperturbed "linear" state [say, $\left(n_{0}, l_{0}, m_{0}\right)$ th one]. Gathering the terms of the first order with respect to $\epsilon$ expansion we conclude that $\psi_{1}$ can be represented in the form $\left(\mathcal{E}_{0}=\mathcal{E}_{n_{0}, l_{0}, m_{0}}\right)$

$$
\xi_{0}^{(1)}=A\left(\rho_{1}, \ldots ; \tau_{1}, \ldots\right) e^{-i \mathcal{E}_{0} \tau_{0}}\left|n_{0}, l_{0}, m_{0}\right\rangle
$$

Hereafter the notation $\left(\rho_{1}, \ldots ; \tau_{1}, \ldots\right)$ is used in order to indicate the dependence on all spatial and temporal variables, which are slower than those written explicitely [i.e. for example in (3.12) the amplitude depends on $\tau_{1}, \tau_{2}, \ldots .$. .

The solution of the equation appearing in the second order of $\epsilon$ is represented in the form of the expansion

$$
\psi_{2}=\sum_{n, l, m}^{\prime} B_{n^{\prime}}\left(\rho_{1}, \ldots ; \tau_{1}, \ldots\right) e^{-i \mathcal{E}_{0} \tau_{0}}|n, l, m\rangle .
$$

Hereafter a prime means that the sum is computed over all arguments such that $n \neq n_{0}, l \neq l_{0}$, and $m \neq m_{0}$. The respective equation of the second order takes the form

$$
\sum_{n, l, m}^{\prime}\left(\mathcal{E}_{0}-\mathcal{E}_{n, l, m}\right) B_{n, l, m}|n, l, m\rangle+\left[i \frac{\partial A_{n}}{\partial \tau_{1}}+\left(2 \frac{\partial}{\partial \rho_{0}}+\frac{d-1}{\rho_{0}}\right) \frac{\partial A_{n}}{\partial \rho_{1}}\right]\left|n_{0}, l_{0}, m_{0}\right\rangle=0
$$

Applying $\left\langle n_{0}, l_{0}, m_{0}\right|$ to this equation (see Appendix) one arrives at the relation $\partial A / \partial \tau_{1}=0$. Here we use the property 


$$
\left(\xi_{n}, \hat{p} \xi_{n}\right)=i \int_{0}^{\infty} \xi_{n}\left(\rho_{0}\right)\left(2 \frac{\partial}{\partial \rho_{0}}+\frac{d-1}{\rho_{0}}\right) \xi_{n}\left(\rho_{0}\right) \rho_{0}^{d-1} d \rho_{0}=0
$$

with

$$
\hat{p}=i\left(2 \frac{\partial}{\partial \rho_{0}}+\frac{d-1}{\rho_{0}}\right)
$$

being the operator of the radial component of the (dimensionless) linear momentum. Then one concludes that $A$ does not depend on $\tau_{1}, A \equiv A\left(\rho_{1}, \ldots ; \tau_{2}, \ldots\right)$.

The coefficient $B_{n_{0}, l_{0}, m_{0}}$ is equal to zero identically [otherwise it could be made zero by simple renormalization of initial conditions for (2.9) ]. In order to find other coefficients $B_{n, l, m}$ we compute the inner product with $\left\langle n_{0}, l_{0}, m_{0}\right|$. Introducing matrix elements

$$
p_{n^{\prime} n}=\left(\xi_{n}, \hat{p} \xi_{n^{\prime}}\right)
$$

corresponding to the transition between the states $n^{\prime}$ and $n$, originated by the radial component of the linear momentum considered as a perturbation, we obtain the coefficients of the second order term

$$
B_{n, l, m}\left(\rho_{1}, \ldots ; \tau_{2}, \ldots\right)=i \frac{p_{n_{0} n}}{\mathcal{E}_{0}-\mathcal{E}_{n, l, m}} \frac{\partial A}{\partial \rho_{1}}
$$

Finally, collecting all the terms of the third order of $\epsilon$ and using the explicit form of the second order addendum found above we arrive at the dynamical equation for the amplitude $A$

$$
i \frac{\partial A}{\partial \tau_{2}}+\mathcal{D}_{0} \frac{\partial^{2} A}{\partial \rho_{1}^{2}}-\chi_{n_{0} n_{0}}|A|^{2} A=0
$$

Here

$$
\mathcal{D}_{0}=\mathcal{D}_{n_{0}, l_{0}, m_{0}}=1-\sum_{n, l, m}^{\prime} \frac{\left|p_{n_{0} n}\right|^{2}}{\mathcal{E}_{n, l, m}-\mathcal{E}_{0}}
$$

is the effective dispersion and

$$
\chi_{n n^{\prime}}=\int \xi_{n}^{2}(\rho) \xi_{n^{\prime}}^{2}\left(\rho^{\prime}\right) U\left(\left|\tilde{\mathbf{r}}-\tilde{\mathbf{r}}^{\prime}\right|\right) d \tilde{\mathbf{r}} d \tilde{\mathbf{r}}^{\prime}
$$

is the effective nonlinearity. As a matter of fact the effective nonlinearity can be computed under the assumption that the characteristic scale of the ground state is much larger than the width of the kernel $U(|\tilde{\mathbf{r}}|)$ of the interaction potential. Taking into account (2.7), (3.1), and (A1), (A3) one concludes that this supposition is valid with great accuracy for several lowest eigenstates $\xi_{n}(\rho)$. Then $U(|\mathbf{r}|)$ in the integrand of $(3.20)$ can be substituted by the delta function and thus $\chi_{n n}=\left(\xi_{n}^{2}, \xi_{n}^{2}\right)$.

In the explicit form of $\mathcal{D}_{0}$ one can recognize the second order addendum to the energy of an effective particle induced by the perturbation operator $\hat{p}$. Thus the effective dispersion has a contribution of the probability of the energy transfer among the levels in the confining potential. Notice that the mentioned transition occur among levels with the same orbital and azimutal quantum numbers (in the case at hand they are equal to zero).

Equation (3.18) is considered on the semiline $\left(\rho_{1} \geq 0\right)$ subject to the boundary condition $\lim _{\rho_{1} \rightarrow \infty} A\left(\rho_{1}, \ldots ; \tau_{2}, \ldots\right)=$ $\mathcal{A} e^{i \tilde{\omega} \tau_{2}}$ where the constant $\mathcal{A}$ can be interpreted as an amplitude of the fundamental state of the condensate: after all it must be found from the normalization condition. [Notice that other boundary conditions, of less practical interest, are also compatible with the problem at hand].

Let us now return to the discussion of the main scales of the problem. In the dimensionless variables the spatial scale of the amplitude $A\left(\rho_{1}, \ldots ; \tau_{2}, \ldots\right)$ can be estimated as $1 / \epsilon$. On the other hand the problem possess another important dimensionless scale $1 / \sqrt{\Omega}$ which determines the wave function localization due to the confining potential. An interesting and physically relevant situation appears when $\epsilon \gg \sqrt{\Omega}$, what in physical variables means $8 \pi N a_{s} / a_{0} \gg 1$ [28], a condition which is satisfied in current experimental setups. The last requirement can be understood equivalently as smallness of the healing length $\xi$ compared with the linear oscillator length $\xi \ll a_{0}$. In this situation the excitation width is much smaller than the volume "available" for the condensate and in the case of positive scattering length one can obtain localized excitations against the background. This is the regime of validity of our theory as has been put in more mathematical terms in the first part of this section. 


\section{APPLICATION: VORTEX SHAPE AND STABILITY}

One of the simplest excitations of the ground state condensate one can construct have the form of static "dark" soliton solutions of Eq. (3.18), i. e. "black holes" or vortices (when a local phase is added). Joining the different contributions from the multiscale method we obtain

$$
\Psi=\frac{1}{\sqrt{\pi}} \frac{a_{s}}{\xi} \exp \left[-i\left(\frac{2}{a_{0}^{2}}+\frac{1}{a_{z}^{2}}+8 \pi \sqrt{\frac{n}{a_{s}}}\right) \frac{\hbar t}{2 m}\right] \exp \left(-\frac{r_{\perp}^{2}}{2 a_{0}^{2}}-\frac{z^{2}}{2 a_{z}^{2}}\right) \tanh \left(\frac{r_{\perp}}{\sqrt{2 \mathcal{D}_{0}} \xi}\right) e^{i m \phi}+O\left(n a_{s}^{3}\right) .
$$

This solution approximates the shape of a vortex line with axis of rotation $z$ in trap of transverse size $a_{0}$.

This is not the only solution one can construct, as an example we present also a solution corresponding to a "black hole" in three dimensions

$$
\Psi=\frac{1}{\pi^{3 / 4}} \frac{a_{s}}{\xi} \exp \left[-i\left(\frac{3}{a_{0}^{2}}+2^{7 / 2} \sqrt{\frac{\pi n}{a_{s}}}\right) \frac{\hbar t}{2 m}\right] \exp \left(-\frac{r^{2}}{2 a_{0}^{2}}\right) \tanh \left(\left(\frac{2}{\pi}\right)^{1 / 4} \frac{r}{\sqrt{2 \mathcal{D}_{0}} \xi}\right)+O\left(n a_{s}^{3}\right)
$$

where $r=(x, y, z)$.

In the above formulas we have neglected the difference between $\lambda$ and $a_{s}$ [see (2.7)]. They however still contain the quantity $\mathcal{D}_{0}$ to be estimated. To this end we employ $(\mathrm{A} 1)$ and $(\mathrm{A} 3)$ and compute $(3.19)$ [29]. The result is $\mathcal{D}_{0} \approx 0.586$ and $\mathcal{D}_{0} \approx 0.728$ for (4.1) and (4.2) respectively. Thus in the physical units the effective width of the black hole can be estimated to be of order of the healing lengths which is the expected result for the vortex size.

It must be said that the result naturally does not depend on $\Omega$, a fact which is explained by taking into account that in the parabolic trap the energy levels are equidistant. Another point to be mentioned is that as a matter of fact we have computed the terms of order of $O\left(n a_{s}^{3}\right)$. They are given by (3.13), (3.17) and are not represented explicitly because of cumbersome form. In other words the theory provides one with the BEC wavefunction computed with accuracy $O\left(\left(n a_{s}^{3}\right)^{3 / 2}\right)$ to problems whose number of particles is not too large.

One of the advantages of the multiscale method is that it guarantees the dynamical stability of the solution up to a certain time scale. In order to explain this last point we notice that the method is based on eliminating the secular terms in the third order with respect to the small parameter. This in particular means that the characteristic time of evolution of instabilities of the solutions (if any) is given by $\epsilon^{3} \tau$. The main implication is that the instabilities against the background solution (which is the vortex in the case at hand) cannot develop up to $\tau \sim \epsilon^{-3}$. Recalling now (3.11) and returning to the physical variables we obtain that for the typical values of $\epsilon \sim 10^{-2}$ any instability could develop only for times larger than 10 seconds, which is of the order of the life-time of the condensate. In fact, this prediction is consistent with the previous numerical observation 22] that the vortex is a stable object.

This result is relevant since a mathematical proof of the expected dynamical stability of a vortex in a trap (i.e. when dissipation is zero) is not available yet. However, the numerical simulations (which are also valid only for limited times) point that a vortex should be a dynamically stable object. The analysis of the linearized operator around the vortex done in Ref. [22] was not completely conclusive since there were some zero eigenvalues which could lead to instabilities mediated by high order terms in the linearized evolution operator. In this sense the multiscale technique adds a new argument in favor of long time stability of the vortex in the framework of the mean field modelization of the phenomenon.

We remark that the previous discussion applies to dynamical stability, i. e. stability of the solution under perturbations on the initial data. It was discussed on the previous works on vortex stability that the system is energetically unstable [21,22], while the question of its Lyapunov stability remains open. Our analysis concerns only the subject of dynamical stability and confirm the numerical observations by many authors that vortices in a trap are very long lived and could even be completely stable [22].

\section{CONCLUSION}

In the present paper we have developed the multiscale approach for obtaining explicit (approximate) solutions to the GP equation for condensates with sufficiently small number of particles which are characterized by fast spatial scales of the order of the healing length, and thus cannot be described within the framework of the conventional hydrodynamical approach. One of the advantages of the method is that it guarantees the stability of the solution as discussed in Sec. [IV which allows us to put lower bounds on the time a vortex survives under possible dynamical instabilities described within the framework of the GP equation.

Another outcome of the theory developed above is that the solutions of the healing length size cannot be excited only as a background state. Some accompanion modes, corresponding to higher levels of the confining potential are 
excited simultaneously. All these contributions are included in the constants $\mathcal{D}_{0}$ appearing in the reduced equations (3.18).

One more advantage of the multiscale method is that, by reducing the problem to solving the effective one dimensional nonlinear Schrödinger equation, one gets a powerful tool for obtaining other more complicated solutions and even interactions among them, a point which will be the subject of further study.

The present paper provides a new analytical tool which we hope will be of interest for theoretical progresses in the analysis of BEC problems.

\section{ACKNOWLEDGMENTS}

We are in debt to G. Alfimov for many fruitful discussions. V. V. K. is grateful to University of Castilla-La Mancha for warm hospitality. V.M.P-G. has been supported by DGCYT under grant PB96-0534. The cooperative work has been supported through the bilateral program DGCyT-HP1999-019/Ação No E-89/00.

\section{APPENDIX A: EIGENFUNCTIONS}

For the sake of convenience here we present the orthonormalized eigenfunctions. In the case of potential with cilindrical symmetry they are

$$
\begin{gathered}
\xi_{n, m}(\rho)=\sqrt{2 \Omega \frac{n !}{(n+m) !}}(\sqrt{\Omega} \rho)^{m} e^{-\Omega \rho^{2} / 2} L_{n}^{(m)}\left(\Omega \rho^{2}\right), \\
\zeta_{l, m}(\phi, z)=\frac{\Omega_{z}^{1 / 4}}{\pi^{3 / 4} \sqrt{2^{l+1} l !}} e^{i m \phi} e^{-\Omega_{z} z^{2} / 2} H_{l}\left(\sqrt{\Omega_{z}} z\right) .
\end{gathered}
$$

Here $H_{n}(\cdot)$ is the Hermite polinomial and $L_{n}^{(m)}(\cdot)$ is the Laguerre polinomial. When spherical symmetry is imposed the eigenfunctions are given by

$$
\begin{gathered}
\xi_{n, l}(\rho)=2\left[\frac{2^{n+l}}{(2(n+l)+1) ! !} \Gamma(n+1)\right]^{1 / 2}\left(\frac{\Omega^{3}}{\pi}\right)^{1 / 4} e^{-\Omega \rho^{2} / 2}(\sqrt{\Omega} \rho)^{l} L_{n}^{(l+1 / 2)}\left(\Omega r^{2}\right), \\
\zeta_{l, m}(\theta, \phi) \equiv Y_{l}^{m}(\theta, \phi)=(-1)^{m+|m|} i^{l}\left[\frac{2 l+1}{4 \pi} \frac{(l-|m|) !}{(l+|m|) !}\right]^{1 / 2} P_{l}^{|m|}(\cos \theta) e^{i m \phi} .
\end{gathered}
$$

The inner product in this space is defined by

$$
\left\langle n, l, m \mid n^{\prime}, l^{\prime}, m^{\prime}\right\rangle=\int_{-\infty}^{\infty} d z \int_{0}^{2 \pi} d \phi \int_{0}^{\infty} d \rho \rho \bar{\xi}_{n, m}(\rho) \xi_{n^{\prime}, m^{\prime}}(\rho) \bar{\zeta}_{l, m}(z, \phi) \zeta_{l^{\prime}, m^{\prime}}(z, \phi)
$$

in the case of cilindrical symmetry and by

$$
\left\langle n, l, m \mid n^{\prime}, l^{\prime}, m^{\prime}\right\rangle=\int_{-\pi}^{\pi} d \theta \int_{0}^{2 \pi} d \phi \int_{0}^{\infty} d \rho \rho \bar{\xi}_{n, m}(\rho) \xi_{n^{\prime}, m^{\prime}}(\rho) \bar{\zeta}_{l, m}(\theta, \phi) \zeta_{l^{\prime}, m^{\prime}}(\theta, \phi)
$$

in the case of spherical simmetry

[1] M. H. Anderson, J. R. Ensher, M. R. Matthews, C. E. Wieman, E. A. Cornell, Science. 269, 198 (1995); K. B. Davis, M.-O. Mewes, M. R. Andrews, N. J. van Druten, D. S. Durfee, D. M. Kurn, W. Ketterle, Phys. Rev. Lett. 75 (1995) 3969 ; C. C. Bradley, C. A. Sackett, J. J. Tollett, R. G. Hulet, ibid., 75, 1687 (1995). 
[2] See e.g. the reference list on the BEC homepage http://amo.phy.gasou.edu/bec.htm]

[3] A. Griffin, Phys. Rev. B. 539341 (1996).

[4] M. Holland, J. Cooper, Phys. Rev. A 531954 (1996).

[5] S. Stringari, Phys. Rev. Lett. 77, 2360 (1996); M. Edwards, P. A. Ruprecht, K. Burnett, R. J. Dodd and Charles W. Clark ibid. 77, 1671 (1996); Y. Castin and R. Dum, ibid. 77, 5315 (1996); V. M. Pérez-García, H. Michinel, J. I. Cirac, M. Lewenstein, P. Zoller, ibid. 775320 (1996).

[6] Yu Kagan, A. E. Muryshev, and G. V. Shlyapnikov, Phys. Rev. Lett 81, 993 (1998); V. M. Pérez-García, H. Michinel, J. I. Cirac, M. Lewnstein, P. Zoller, Phys. Rev. A 561424 (1997); T. Tsurumi and M. Wadati, J. Phys. Soc. Jpn. 66, 3035 (1998).

[7] H. Michinel, V. M. Pérez-García, R. de la Fuente, Phys. Rev. A 601513 (1999).

[8] B. Esry, C. Greene, J. Burke, J. Bohn, Phys. Rev. Lett. 78 (1997) 3594; T. Busch, J. I. Cirac, V. M. Perez-Garcia, P. Zoller, Phys. Rev. A 56 (1997) 2978; R. Graham, D. Walls, Phys. Rev. A 57 (1998) 484; B. Esry, C. Greene, ibid. 57 (1998) 1265; H. Pu, P. Bigelow, Phys. Rev. Lett. 80 (1998) 1130; B. Esry, C. Greene, Nature 392 (1998) 434; J. Burke, J. Bohn, B. Esry, C. Greene, Phys. Rev. Lett. 80 (1998) 2097; H. Law, H. Pu, P. Bigelow, ibid. 81 (1998) 5257; T. Isochima, K. Machida, T. Ohni, Phys. Rev. A 604857 (1999).

[9] J. Williams and M. Holland, Nature (London) 401, 568 (1999); M. R. Matthews, B. P. Anderson, P. C. Haljan, C. E. Wiemann, E. A. Cornell, Phys. Rev. Lett. 83 (1999) 2498.

[10] J. J. García-Ripoll, V. M. Pérez-García, http://xxx.lanl.gov/abs/cond-mat/9910015, (submitted to Phys. Rev. Lett.); V. M. Pérez-García, J. J. García-Ripoll, http://xxx.lanl.gov/abs/cond-mat/9912308, (submitted to Phys. Rev. A)

[11] M. R. Andrews et al., Phys. Rev. Lett. 79 (1997); G. M. Kavoulakis, C. Petick, Phys. Rev. A 58 1563 (1998); E. Zaremba, Phys. Rev. A 57 (1998) 517; T. Tsurumi, M. Wadati, Jour. Phys. Soc. Jpn. 67 (1998) 2294; A. D. Jackson, G. M. Kavoulakis, C. Petick, Phys. Rev. A 58 (1998) 2417; M. Flieser, R. Graham, ibid. 59 (1999) R27; O. Zobay, S. Potting, P. Meystre, E. Wright, Phys. Rev. A 59 (1998) 643; S. Burger, K. Bongs, S. Dettmer, W. Ertmer, K. Sengstock, A. Sanpera, G. V. Shlyapnikov, M. Lewenstein, Phys. Rev. Lett. 835198 (1999).

[12] L. Vázquez, L. Streit, V. M. Pérez-García, Eds., Nonlinear Klein-Gordon and Schrödinger Systems: Theory and Applications, World Scientific (1996)

[13] C. Sulem, P. Sulem, The Nonlinear Schrödinger Equation, Springer-Verlag, Berlin (1999).

[14] B. D. Fried, Y. H. Ichikawa Y. H., Jour. Phys. Soc. Jpn. 34, 1073 (1973)

[15] A. Hasegawa, Optical Solitons in Fibers, (Springer-Verlag, Berlin, 1989).

[16] A. C. Newell, J. V. Moloney, Nonlinear Optics, Addison-Wesley (1992).

[17] R.K. Dodd, J.C. Eilbeck, J.D. Gibbon, and H.C. Morris, "Solitons and nonlinear wave equations", Academic Press (1982).

[18] A.S. Davydov, Solitons in Molecular Systems (Reidel, Dordrecht, 1985).

[19] J. L. Rosales, J. L. Sánchez-Gómez, Phys. Lett. A 66 (1992) 111

[20] V. M. Pérez-García, H. Michinel, and H. Herrero, Phys. Rev. A 57, 3837 (1998)

[21] S. Stringari, Phys. Rev. Lett. 77, 2360 (1996); D. Rokhsar, Phys. Rev. Lett. 79, 2164 (1997); R. J. Dodd, K. Burnett, M. Edwards, and C. W. Clark, Phys. Rev. A 56, 587 (1997); T. Isoshima and K. Machida, J. Phys. Soc. Jpn. 66, 3502 (1997); A. A. Svidzinsky, A. L. Fetter, Phys. Rev. A 58, 3168 (1998); A. L. Fetter, J. Low Temp. Phys. 113, 189 (1998); T. Isoshima and K. Machida, J. Phys. Soc. Jpn. 68, 487 (1999); T. Isoshima and K. Machida, Phys. Rev. A 59, 2203 (1999)

[22] J. J. García-Ripoll, V. M. Pérez-García, Phys. Rev. A 604864 (1999).

[23] V. M. Pérez-García, V. V. Konotop, J. J. García-Ripoll, (submitted to Phys. Rev. E).

[24] F. Dalfovo, S. Giorgini, L. P. Pitaevski, S. Stringari, Rev. Mod. Phys. 71463 (1999).

[25] L. D. Landau and E. M. Lifshitz Quantum Mechanics. Nonrelativistic Theory. (Pergamon Press, New York, 1977

[26] Handbook of Mathematical Functions. Eds M. Abramowitz and I. A. Stegun, (New York, 1964).

[27] K. B. Davis, M.-O. Mewes, M. R. Andrews, N. J. van Druten, D. S. Durfee, D. M. Kurn, and W. Ketterle, Phys. Rev. Lett. 75, 3969 (1995)

[28] It is interesting to mention that this inequality can be written down as $N \gg N_{c}$ where $N_{c}=O\left(\frac{a_{0}}{8 a_{s}}\right)$. This last critical number appears naturally in theory of BEC with attractive interactions. In particular, an opening of a "black hole" in that case at $n>N_{c}$ has been reported recently by M. Ueda and K. Huang http://xxx.lanl.gov/abs/cond-mat/9807359.

[29] The computation have been made with the MAPLE's codes for the Laguerre. In the $2 d$ and $3 d$ cases the number of sum entries computed were 100. However the accuracy of oder of $1 \%$ is achieved by taking into account 30 first terms of both sums. 